\title{
Time - triggered Ethernet Modeling and Evaluation Based on DSPN Qi-feng Ren ${ }^{1, b}$, Guo-quan Zhang ${ }^{1}$, Qi-ming Yang ${ }^{2}$, Jian-dong Zhang ${ }^{2, a}$, Guo-wen $\mathrm{Hu}^{2}$ \\ ${ }^{1}$ Aeronautical radio electronics research institute of China, Shanghai, 200241, China \\ ${ }^{2}$ Northwestern Polytechnical University, Xi'an, 710072, China \\ aemail: jdzhang@nwpu.edu.cn, bemail: ren_qifeng@careri.com
}

Keywords: Time-Triggered Ethernet; TTE; DSPN; Performance Evaluation

\begin{abstract}
The traditional on-board data bus has been unable to meet the characteristics of modern advanced avionics communications equipment. Time-Triggered Ethernet (TTE) has the advantages of real-time, determinism and reliability and can fully adapt to distributed synthesis Modular avionics architecture development. It is of theoretical significance to analyze the bus load and transmission delay in the network by establishing the message transmission model of time-triggered Ethernet with deterministic and stochastic Petri Nets (DSPN).
\end{abstract}

\section{Introduction}

The avionics system has gone through three stages of separation, integration and integration, and is moving towards the "advanced and integrated" stage proposed by the fourth generation avionics system. . With the development of next generation aircraft, The traditional MIL-STD-1553B bus can not meet the data throughput, data transmission rate and reliability requirements of the integrated avionics system.

Time-triggered Ethernet TTE (Time-Triggered Ethernet) has high-speed, high real-time property and reliability, which can meet the avionics system for high-speed, high real-time and reliability performance requirements. So it is qualified to take the place of modern aircraft data bus[1]. At present, TTE has been applied to the actual project, NASA launched the Mars probe by use of the TTE bus, TTE Orion spacecraft (the new generation of space shuttle) backbone. TTE has been used in a series of aerospace projects, and its applications in rail transportation, medical technology, robotics and other related fields are also underway. The application of these areas have a common requirement on network technology: high availability, robustness and time determinism, a unified network can simultaneously support different communication services, low cost and standardization.

TTE networks need to schedule three types of data, such as TT (Time-Triggered), RC (Rate-Constrained) and BE (Best-Effort). In this paper, The generation and transmission of the TTE network are analyzed. A message transmission model is established by using DSPN. The calculation method of the two performance indexes of TTE network is presented. In section 1, this paper introduces the topology structure and time trigger mechanism of TTE bus. In section 2, three kinds of messages are established according to the rules of TT data, RC data and BE data. DSPN model, and then establish a reasonable scheduling mechanism according to the priority of three kinds of message transmission. Finally, these sub-models are combined to establish the DSPN model of TTE network.

\section{Introduction to TTE Bus}

\subsection{Topology Structure Based on TTE}

The TTE bus consists of three types of terminals: Synchronous Controller (SM), Synchronous Client (SC) and Centralized Controller (CM). Under normal circumstances, there is only one CM in the TTE network, and the other is SM or SC. Because there are many terminals in avionics network, each terminal node acts as SM and TTE switch as CM. The avionics network studied in this paper 
has only one switch with no SC.

As shown in Figure 1, this structure is common in ordinary Ethernet connection. End system must be communicated through the TTE switch, which is easy to centralized control, and the individual terminal failure can not affect the normal communication of other terminals. However, the most disadvantage of this structure is that TTE switches must have a high reliability, because if the TTE switch failure, the entire system will be paralyzed. So the system has two TTE switches, one as a backup.

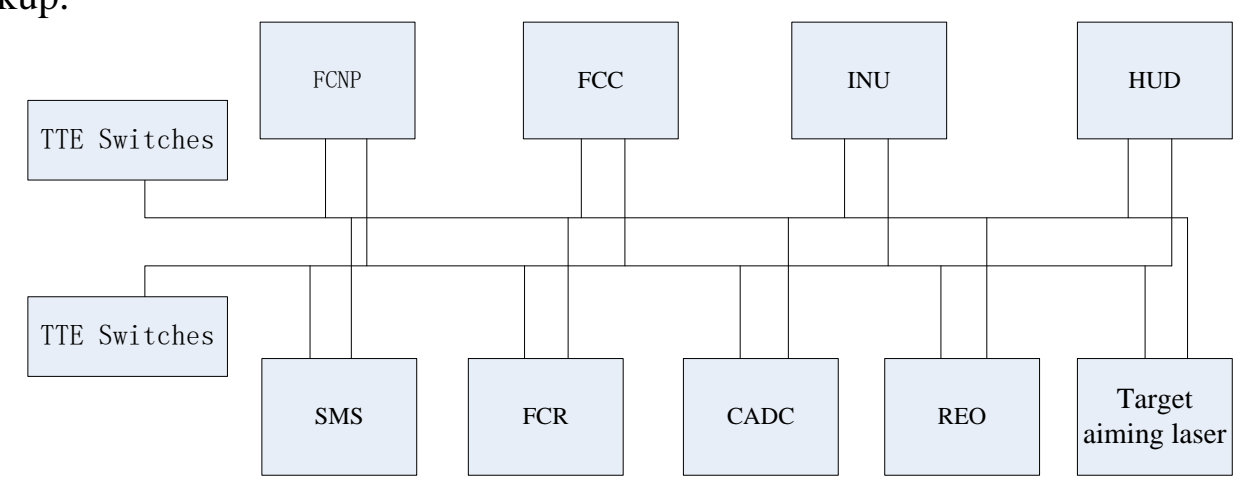

Figure1. TTE avionics network topology

\subsection{Time-triggered service mechanism}

1) Clock synchronization mechanism

TTE network clock synchronization is divided into two steps, as shown in Figure 2.
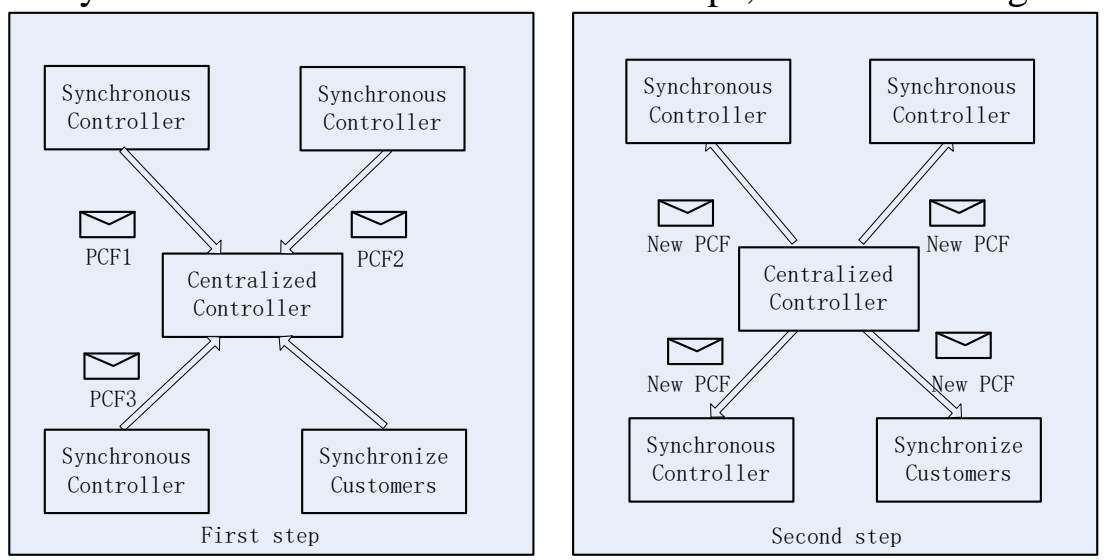

Figure 2. TTE clock synchronization process [3]

In the first step, each synchronization controller sends a Protocol Control Frame (PCF) to the central controller. The PCF frame can not be transmitted at any time, the synchronization controller sends the time control frame (PCF) after the local clock reaches a certain time. The PCF frame is related to the local clock of the synchronous controller, such as the transmission time. When a PCF frame arrives at the centralized controller via a link, the PCF frame records the delays experienced during propagation, such as propagation delay, dynamic transmission delay, and dynamic reception delay [3].

In the second step, after receiving the different PCF frames (PCF1-3) on the link, the centralized controller obtains a new PCF frame through the sequence keeping algorithm and the centralized algorithm, and then sends the frame to the synchronization Customers and synchronization controllers. The centralized controller acts as an arbitration mechanism, calculates a clock based on the PCF frame sent by the synchronization controller, and then sends the message back to the synchronization controller and the synchronization client, The synchronization controller and the synchronization client modify the local clock according to the new PCF frame until the global synchronization is completed [3].

2)TTE message transmission mechanism

The LTE protocol supports the transmission of three different messages:

1) TT message: The transmission of TT message using preemption mechanism, when the bus is occupied, TT messages to seize the transmission of other information resources to ensure their 
transmission time Certainty.

2) RC message: The RC message is also generated by the synchronous client or the synchronous controller, but its transmission is independent of time and can occur at any time. In the transmission process, its transmission rate is limited, its transmission rate can be limited through the token bucket mechanism, which can guarantee a certain transmission delay and jitter.

3) BE messages: Traditional Ethernet messages. In the TTE network, all terminals can send BE messages at any time, the transmission rate is not limited and is only influenced by the communication bandwidth, the message is the largest network transmission data, General network congestion is caused by the BE data.

In order to guarantee the transmission delay of TT messages, the priority of TT messages is defined as follows: TT message is highest, $\mathrm{RC}$ message is next, $\mathrm{BE}$ message is the lowest. The following describe the transmission characteristics of the three messages in detail.

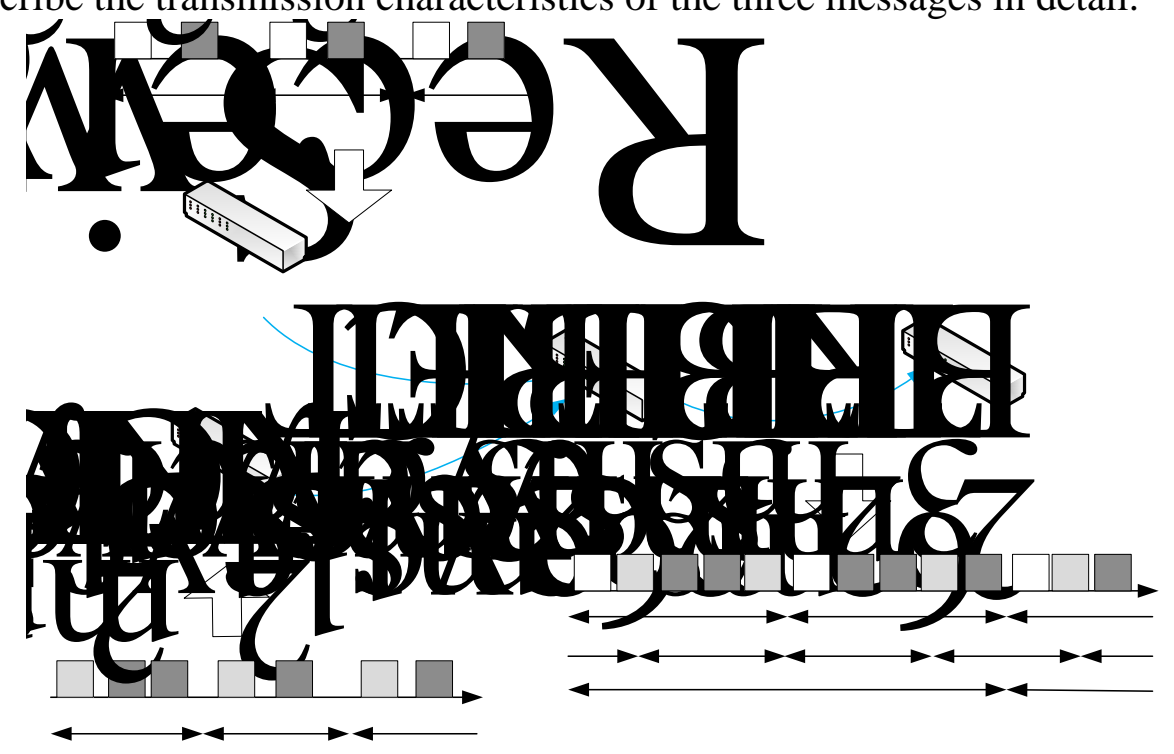

Figure 3. TTE message transmission diagram [1]

Figure 3 shows the mechanism of message transmission in LTE network, Sender1 sends the message in 3ms period, including periodic TT message and BE message; Sender2 sends periodic TT messages and other ET messages in $2 \mathrm{~ms}$ period. The messages are transmitted to the receiver through the switch according to the transmission mechanism. The transmission order of the messages in the bus is shown in Fig.3 [1].

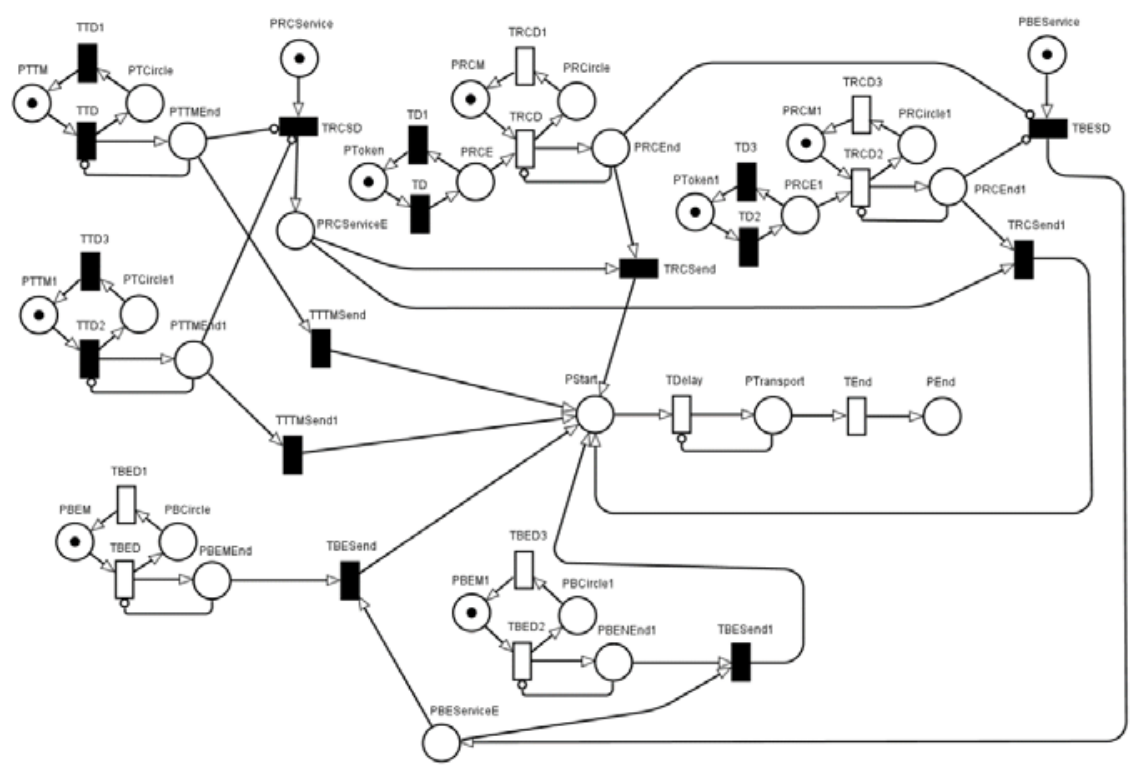

Figure 4. DSPN model of TTE bus 


\section{Time - triggered Ethernet Modeling Based on DSPN}

\subsection{DSPN Model of TTE Bus}

In the TTE bus system model shown in Figure 4, there are two TT messages, two RC messages, and two BE messages. The two TT messages utilize the determination of time transitions TTD, TDD1 and TTD2, TTD3 to cause them to occur at different times. In the process of transmission, two RC messages are first-served, and in the above model, they are simulated by the locations PRCService, PRCServiceE and the time-variant TRCSD. The two groups of BE messages avoid collisions in the same way as the RC messages. The PBEService, PBEServiceE and TBESD are used to simulate the first-come-first-served mechanism. Among the three different messages, TT has the highest priority, RC second, BE the lowest. Position PStart, Transport, PEnd and transitions TDelay, Tend simulate the transmission of messages.

\subsection{DSPN model analysis}

When TT data is generated, set the time to determine the different time to change, so that different TT data is sent terminal in their own set time. In the message transfer model of this paper, make the following settings:

a. TT data transmission period is $40 \mathrm{~ms}$, the send time of two senders is different, TTD is $30 \mathrm{ms,}$ TTD2 is 20ms, and TTD3 is $20 \mathrm{~ms}$.

b. The RC data defines its transmission rate, where it is sent one frame every $20 \mathrm{~ms}$. TD is $10 \mathrm{~ms}$, TD1 is $10 \mathrm{~ms}$, TD2 is $5 \mathrm{~ms}$, and TD3 is $15 \mathrm{~ms}$.

c. The average implementation of random changes in transition settings, the simulation set the Ethernet frame length of 1024 bytes, the link transmission speed of 10Mbit / s. So the transmission of one frame needs $1024 * 8 / 10=0.82 \mathrm{~ms}$.

\subsection{Simulation results analysis}

Figure 5 shows the variation of the bus load in the network. As there are a large number of BC data in the network, there are RC message transmission at $5 \mathrm{~ms}$, TT and RC message transmission at $10 \mathrm{~ms}$, TT message transmission at $20 \mathrm{~ms}$, RC message transmission at $25 \mathrm{~ms}$ and $30 \mathrm{~ms}$.

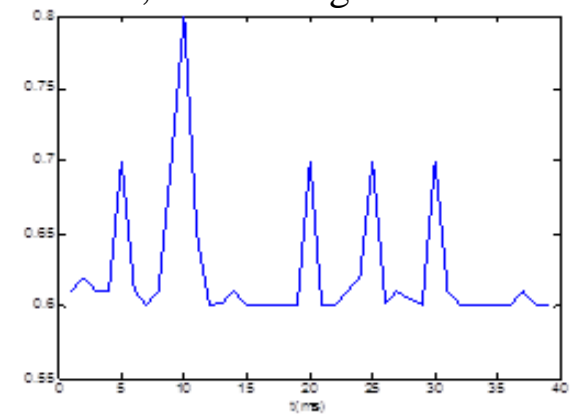

Figure 5. TTE network bus load

The TT message delay time is shown in Figure 6. It can be seen from the figure that TT message delay in the network generates only when the TT message is transmitted.

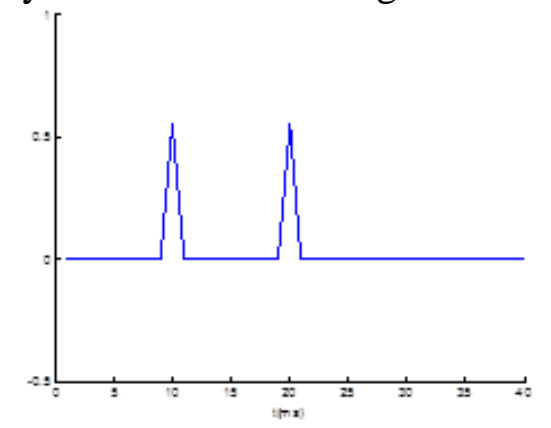

Figure 6. TT message delay time

The RC message delay time is shown in Figure 7. It can be seen from the figure that RC message delays at $5 \mathrm{~ms}, 10 \mathrm{~ms}, 25 \mathrm{~ms}$ and $30 \mathrm{~ms}$. 




Figure 7. RC message delay time

The BE message delay time is shown in Figure 8. The BE message delay will always exist because the $\mathrm{BE}$ data has been present in the network. due to the presence of the $\mathrm{RC}$ and TT messages and the lowest priority of the $\mathrm{BE}$ message, The $\mathrm{BE}$ message delay time increases at time $5 \mathrm{~ms}, 10 \mathrm{~ms}, 20 \mathrm{~ms}, 25 \mathrm{~ms}$, and 30ms.



Figure 8. BE message delay time

\section{Conclusion}

In this paper, the TTE network is modeled from the process of message generation and transmission with DSPN. The method and simulation results of analyzing the TTE network bus load and network delay time are given. As the simulation time advances, the bus load and delay times also change. The results of the analysis can reflect the changes of bus load and transmission delay time. he DSPN model constructed in this paper can show the transmission characteristics of TT, RC and $\mathrm{BE}$ in the network intuitively. It can also analyze the bus load and transmission delay. However it can not make a specific analysis of the property of TTE protocol, only show the abstract performance of the time-triggered network data flow. The core mechanism of the TTE network is not described in detail, and the key TT data in the TTE network is not analyzed in detail. Therefore, this model can not reflect the characteristics of time-triggered network. It is only an abstract description of TTE network to help readers have a general understanding of TTE network.

\section{Acknowledgement}

The work was supported by the Science and Technology on Avionics Integration Laboratory .The gratitude goes to all the staff of the Foundation.

\section{References}

[1] Leen G , Heffernan D. Modeling and verification of a time-triggered networking protocol[C]//Networking, International Conference on Systems and International Conference on Mobile Communications and Learning Technologies, 2006. ICN/ICONS/MCL 2006. International Conference on. IEEE, 2006: 178-178.

[2] Kopetz H, Bauer G. The time-triggered architecture[J]. Proceedings of the IEEE, 2003, 91(1): 112-126.

[3] Kopetz H, Ademaj A, Grillinger $\mathrm{P}$, et al. The time-triggered ethernet (TTE) design[C]//Object-Oriented Real-Time Distributed Computing, 2005. ISORC 2005. Eighth IEEE 
International Symposium on. IEEE, 2005: 22-33. [4] Joerg Christian Wolf, Phil Hall, Paul Robinson, Phil Culverhouse. Bioloid based Humanoid Soccer Robot Design, 2007.

[4] SAE AS6802 Time-triggered Ethernet[S].

[5] ZHANG Jian-dong, WU Yong et al. Performance Analysis of Integrated Avionics Bus System Based on DSPN [J]. Journal of Northwestern Polytechnical University, 2005, 23 (2): 243-248.

[6] WANG Wang, YAO Shu-Zhen et al. Modeling and Performance Analysis of TTE Bus Based on DSPN [J] .Computer Science, 2014, 41 (7): 45-48. 